\title{
Continuous-variable teleportation in the characteristic-function description
}

\author{
Paulina Marian ${ }^{1}$ and Tudor A. Marian ${ }^{2}$ \\ 1 Department of Chemistry, University of Bucharest, \\ Boulevard Regina Elisabeta 4-12, R-030018 Bucharest, Romania \\ 2 Department of Physics, University of Bucharest, \\ P.O.Box MG-11, R-077125 Bucharest-Măgurele, Romania
}

(Dated: July 9, 2018)

\begin{abstract}
We give a description of the continuous-variable teleportation protocol in terms of the characteristic functions of the quantum states involved. The Braunstein-Kimble protocol is written for an unbalanced homodyne measurement and arbitrary input and resource states. We show that the output of the protocol is a superposition between the input one-mode field and a classical one induced by measurement and classical communication. We choose to describe the input state distortion through teleportation by the average photon number of the measurement-induced field. Only in the case of symmetric Gaussian resource states we find a relation between the optimal added noise and the minimal EPR correlations used to define inseparability.
\end{abstract}

PACS numbers: 03.67.-a; 42.50.Dv; 03.65.Ud; 03.67.Mn

\section{INTRODUCTION}

The principal idea of the teleportation process first described in Ref. [1] is that two distant operators, Alice at a sending station and Bob at a receiving terminal, share an entangled bipartite quantum state and exploit its nonlocal character as a quantum resource. In the continuousvariable case, the resource state which is also called an Einstein-Podolsky-Rosen (EPR) state [2] is a two-mode entangled quantum state. The protocol for teleportation of quantum radiation field states originally proposed by Braunstein and Kimble [3] within the Wigner-function formalism was subsequently given in several other descriptions. Wave-function treatments in the Fock-state basis [4], continuous-coordinate representation [5, 6] or in the coherent-state expansion [7] are particularly useful in the pure-state input case and ideally faithful teleportation. Note also the formalism of the transfer operator put forward in Ref. [8]. Nonideal teleportation was treated by considering either a mixed entangled two-mode state 9] shared by the two distant partners Alice and Bob or imperfect Bell measurements [3, 7]. Phase-space descriptions were given using the $P$-representation of the density operator in a treatment valid also for mixed-state input [10]. The teleportation fidelity of mixed one-mode nonclassical Gaussian states through a symmetric channel was quantified in our work Ref. 11] by using the Uhlmann fidelity. A relation between the entanglement of the symmetric resource state measured by Bures distance 12 and the accuracy of teleportation was then analyzed. The Wigner function was used in describing teleportation as a general conditional measurement 13. or to extend the protocol to non-Gaussian states in Ref. 14]. A lot of work was paid to analyze realistic ideas of increasing the teleportation fidelity. A recent survey on these issues is the e-print 15]. The role of teleportation in the continuous-variable quantum information is analyzed in the review Ref. [16]. Especially related to our present work are several papers investigating the possibility of increasing the quality of teleportation by local interventions of the two operators 17, 18, 19]. By changing the protocol, in Ref. 20], the quantum nondemolition interaction is used to obtain entangled states for teleportation. There the incoming field and the two-mode resource field are superposed at an unbalanced beam splitter and the teleportation accuracy is discussed in terms of added noise to the input field. Note finally that in the recent e-print 21] the teleportation protocols were efficiently included in a more general evaluation of the capacity of Bosonic channels.

In the present paper we give a description of the continuous-variable teleportation protocol [3] in terms of the characteristic functions (CF's) of the quantum states involved. Our main tool in deriving the $\mathrm{CF}$ of the teleported state is the Weyl expansion of the density operator. In Sec. II we consider the Braunstein-Kimble protocol written for an unbalanced homodyne measurement and arbitrary input and resource states. We show that the $\mathrm{CF}$ of the teleported state is the product between the $\mathrm{CF}$ of the input state and a function only depending on the properties of the two-mode resource state and the geometry of measurement. We identify this function as the normally ordered CF of a measurement-induced field. In Sec. III we concentrate on the teleportation of an arbitrary one-mode state through a Gaussian channel and discuss the properties of the measurement-superposed field state. We show that this is a classical state, namely it possesses a well defined $P$-representation. We choose to describe the input state distortion through teleportation by the average photon number of the measurementcreated field. In Sec.IV we analyze the possibility of minimizing this variable by local interventions on the resource state. We conclude by elaborating on the recently found relation between optimal fidelity of teleportation and the entanglement of the resource state [19]. 


\section{UNBALANCED HOMODYNE MEASUREMENT}

The density operator of the input one-mode state $\rho_{\text {in }}$ (to be teleported) and that of the two-mode state $\rho_{A B}$ shared by Alice and Bob can be written as Weyl expansions

$$
\rho_{i n}=\frac{1}{\pi} \int \mathrm{d}^{2} \lambda \chi_{i n}(\lambda) D(-\lambda)
$$

and

$\rho_{A B}=\frac{1}{\pi^{2}} \int \mathrm{d}^{2} \lambda_{1} \mathrm{~d}^{2} \lambda_{2} \chi_{A B}\left(\lambda_{1}, \lambda_{2}\right) D_{1}\left(-\lambda_{1}\right) D_{2}\left(-\lambda_{2}\right)$,

where $D(\alpha)=\exp \left(\alpha a^{\dagger}-\alpha^{*} a\right)$ is a Weyl displacement operator and $a$ denotes the annihilation operator. Generally, the Weyl expansion of a Hilbert-Schmidt operator such as the density operator is a powerful tool in quantum optics and points out the well known oneto-one correspondence between the density operator of a $n$-mode field state $\rho$ and its $\mathrm{CF} \chi\left(\lambda_{1}, \lambda_{2} \cdots \lambda_{n}\right):=$ $\operatorname{Tr}\left[\rho D\left(\lambda_{1}\right) D\left(\lambda_{2}\right) \cdots D\left(\lambda_{n}\right)\right]$. We consider that initially the overall state of the system is the three-mode product state $\rho_{i n} \otimes \rho_{A B}$ and adopt the philosophy of the original teleportation idea [1]: the input state in remains unknown to Alice. For the moment we also assume that the states $\rho_{i n}$ and $\rho_{A B}$ are arbitrary. In the first stage of the protocol, by using an unbalanced beam splitter with the rotation angle $\theta$ Alice measures simultaneously the variables

$$
\hat{q}=\cos \theta \hat{q}_{i n}-\sin \theta \hat{q}_{A}, \quad \hat{p}=\sin \theta \hat{p}_{i n}+\cos \theta \hat{p}_{A},
$$

where

$$
\hat{q}_{j}=\left(\hat{a}_{j}+\hat{a}_{j}^{\dagger}\right) / \sqrt{2}, \quad \hat{p}_{j}=\left(\hat{a}_{j}-\hat{a}_{j}^{\dagger}\right) /(\sqrt{2} i)
$$

are the canonical operators and $\hat{a}_{j}$ and $\hat{a}_{j}^{\dagger}, j=1,2$, are the amplitude operators of the modes. A common eigenfunction of the pair of continuous quantum variables $\{\hat{q}, \hat{p}\}$ can be best written in the coordinate representation as the continuous expansion [22]

$$
\begin{aligned}
|\Phi(q, p)\rangle= & \frac{1}{\sqrt{2 \pi} \cos \theta} \int_{-\infty}^{\infty} \mathrm{d} \xi \exp (i p \xi / \cos \theta)|\xi\rangle_{A} \\
& \otimes\left|\xi \tan \theta+\frac{q}{\cos \theta}\right\rangle_{i n},
\end{aligned}
$$

where $(q, p)$ is the outcome of the homodyne measurement. The distribution function of the outcomes predicted by quantum mechanics is

$\mathcal{P}(q, p)=\operatorname{Tr}_{i n, A B}\left\{\left[|\Phi(q, p)\rangle\langle\Phi(q, p)| \otimes I_{B}\right]\left(\rho_{i n} \otimes \rho_{A B}\right)\right\}$.

As a consequence of the nonlocal character of quantum mechanics, the mode B at Bob's remote side is projected onto the state

$\rho_{B}(q, p) \sim \operatorname{Tr}_{i n, A}\left\{\left[|\Phi(q, p)\rangle\langle\Phi(q, p)| \otimes I_{B}\right]\left(\rho_{i n} \otimes \rho_{A B}\right)\right\}$.
Our point here is to provide a formula for the $\mathrm{CF}$ $\chi_{B}\left(\lambda_{2}, q, p\right)$ of the state (2.7) via the Weyl expansions (2.1) and (2.2). To get this, we have first derived the following trace-formula

$$
\begin{aligned}
\operatorname{Tr}\left[|\xi\rangle\left\langle\xi^{\prime}\right| D(-\lambda)\right]= & \exp [i \Im m(\lambda)(\Re e(\lambda)-\sqrt{2} \xi)] \\
& \times \delta\left[\xi^{\prime}-\xi+\sqrt{2} \Re e(\lambda)\right], \quad
\end{aligned}
$$

and applied it to the evaluation of the state (2.7). Then, carefully performed Gaussian integrals led us to

$$
\begin{aligned}
\chi_{B}\left(q, p, \lambda_{2}\right) & =\frac{1}{\mathcal{P}(q, p) \sin (2 \theta)} \frac{1}{\pi^{2}} \\
& \times \int \mathrm{d}^{2} \lambda \exp \left(\mu \lambda^{*}-\mu^{*} \lambda\right) \chi_{i n}(\lambda) \\
& \times \chi_{A B}\left(\cot \theta \Re e \lambda-i \tan \theta \Im m \lambda, \lambda_{2}\right) .
\end{aligned}
$$

Under the integral above, the outcome $(q, p)$ of the measurement is just contained in the displacement parameter

$$
\mu=\frac{1}{\sqrt{2}}\left(\frac{q}{\cos \theta}+i \frac{p}{\sin \theta}\right) .
$$

Equation (2.9) suggests that Bob should perform a displacement of his state with the outcome parameter $\mu$. The communication by classical means between Alice and Bob is thus an important step of the teleportation protocol. As a result of knowing the displacement $\mu$ for every measurement, Bob can finally construct the teleported state through an ensemble averaging with the distribution function (2.6) over all measurement outcomes. Note that with the well known property $\operatorname{Tr}_{B} D_{B}\left(-\lambda_{2}\right)=\pi \delta^{2}\left(\lambda_{2}\right)$ we can readily write the distribution function of the outcomes

$$
\begin{aligned}
\mathcal{P}(q, p)= & \frac{1}{\sin (2 \theta)} \frac{1}{\pi^{2}} \int \mathrm{d}^{2} \lambda \exp \left(\mu \lambda^{*}-\mu^{*} \lambda\right) \chi_{i n}(\lambda) \\
& \times \chi_{A B}(\cot \theta \Re e \lambda-i \tan \theta \Im m \lambda, 0) .
\end{aligned}
$$

The output density operator at Bob's side after the phase-space translation

$$
\rho_{\text {out }}=\iint \mathrm{d} q \mathrm{~d} p \mathcal{P}(q, p) D_{B}(\mu) \rho_{B}(q, p) D_{B}(-\mu),
$$

has a one-mode Weyl expansion as in Eq. 2.1). The weight function of this expansion is the CF of the teleported state which proves to have a remarkably factorized form

$$
\begin{aligned}
\chi_{B, \text { out }}\left(\lambda_{2}\right)= & \chi_{\text {in }}\left(\lambda_{2}\right) \\
& \times \chi_{A B}\left[\Re e\left(\lambda_{2}\right) \cot \theta-i \Im m\left(\lambda_{2}\right) \tan \theta, \lambda_{2}\right] .
\end{aligned}
$$

Equation (2.13) is the central analytic result of the present work 11]. It applies to arbitrary states: mixed 
or pure (Gaussian or non-Gaussian) two-mode resource state $A B$ and classical or nonclassical input state $i n$.

We multiply Eq. (2.13) by $\exp \left(\left|\lambda_{2}\right|^{2} / 2\right)$ and get

$$
\begin{aligned}
\chi_{B, \text { out }}^{(N)}\left(\lambda_{2}\right)= & \chi_{\text {in }}^{(N)}\left(\lambda_{2}\right) \\
& \times \chi_{A B}\left[\Re e\left(\lambda_{2}\right) \cot \theta-i \Im m\left(\lambda_{2}\right) \tan \theta, \lambda_{2}\right],
\end{aligned}
$$

where the superscript $(N)$ stands for normally ordered.

In his seminal paper on the coherent states of the electromagnetic field, Glauber defined the density operator of the superposition of two fields [23]:

$$
\rho_{S}=\int \mathrm{d}^{2} \beta P_{2}(\beta) D(\beta) \rho_{1} D^{\dagger}(\beta) .
$$

In Eq. 2.15), the field (2) is classical having the $P_{2}$ representation and $\rho_{1}$ is the density operator of the field (1). By considering the case in which the field (1) is also classical and described by the $P_{1}$-representation, Glauber obtained the well-known convolution law for a classical superposition:

$$
\begin{aligned}
P_{S}(\beta)= & \int \mathrm{d}^{2} \beta_{1} P_{1}\left(\beta_{1}\right) P_{2}\left(\beta-\beta_{1}\right) \\
& =\int \mathrm{d}^{2} \beta_{2} P_{2}\left(\beta_{2}\right) P_{1}\left(\beta-\beta_{2}\right) .
\end{aligned}
$$

Subsequently, Eq. 2.15 was the starting point in studying the case when the field (1) is allowed to be in a nonclassical state [24, 25, 26]. It was shown that the multiplication law of the normally ordered CF's,

$$
\chi_{S}^{(N)}(\lambda)=\chi_{1}^{(N)}(\lambda) \chi_{2}^{(N)}(\lambda),
$$

derived from $\mathrm{Eq}(2.15)$ is valid only when at least one of the two fields is classical. Actually, in Ref. 24] Kim and Imoto showed that Eq. (2.17) written for two nonclassical fields leads to unphysical results.

Therefore our Eq. (2.14) describes the superposition between the input field in and a one-mode field, say $M$, extracted from the entangled $A B$ field by the measurement performed by Alice followed by the phase-space translation performed by Bob. According to Eq. (2.17) the function $\chi_{A B}\left[\Re e\left(\lambda_{2}\right) \cot \theta-i \Im m\left(\lambda_{2}\right) \tan \theta, \lambda_{2}\right]$ is its normally-ordered CF. Denote its usual CF as

$$
\begin{aligned}
\chi_{M}\left(\lambda_{2}\right)= & \exp \left(-\left|\lambda_{2}\right|^{2} / 2\right) \\
& \times \chi_{A B}\left[\Re e\left(\lambda_{2}\right) \cot \theta-i \Im m\left(\lambda_{2}\right) \tan \theta, \lambda_{2}\right] .
\end{aligned}
$$

Equation (2.18) tells us that the properties of the field state $M$ are fully determined by the two-mode resource state. In the following we focuse on this issue.

\section{PROPERTIES OF THE STATE $M$}

Most of the work on continuous-variable teleportation protocol was done for two-mode Gaussian resources. This is highly motivated by the experimental possibilities of producing entangled states. Originally, the quality of the teleportation protocol was quantified by the overlap of the states in and out for pure states [3, 16], or the Uhlmann fidelity for mixed Gaussian states [1], 28]. So defined, the fidelity of teleportation depends on the input state. Equation (2.13) suggests an alternative way of evaluating the accuracy of the teleportation process by means of the properties of the CF, Eq. (2.18). In the following we consider an arbitrary input state while an undisplaced two-mode Gaussian state is shared by the sender and receiver. Recall that the $\mathrm{CF}$ of an undisplaced two-mode Gaussian state is entirely specified by its covariance matrix $\mathcal{V}$

$$
\chi_{G}(x)=\exp \left(-\frac{1}{2} x^{T} \mathcal{V} x\right),
$$

In Eq. (3.1) $x^{T}$ is a real row vector $\left(x_{1} x_{2} x_{3} x_{4}\right)$. The real, symmetric, and positive $4 \times 4$ covariance matrix $\mathcal{V}$ has the following block structure:

$$
\mathcal{V}=\left(\begin{array}{cc}
\mathcal{V}_{1} & \mathcal{C} \\
\mathcal{C}^{T} & \mathcal{V}_{2}
\end{array}\right)
$$

where $\mathcal{V}_{1}, \mathcal{V}_{2}$, and $\mathcal{C}$ are $2 \times 2$ matrices. Their entries are correlations of the canonical operators $q_{j}=$ $\left(a_{j}+a_{j}^{\dagger}\right) / \sqrt{2}, p_{j}=\left(a_{j}-a_{j}^{\dagger}\right) /(\sqrt{2} i)$, where $a_{j}$ and $a_{j}^{\dagger}$, $(j=1,2)$, are the amplitude operators of the modes. $\mathcal{V}_{1}$ and $\mathcal{V}_{2}$ denote the symmetric covariance matrices for the individual reduced one-mode squeezed thermal states, while the matrix $\mathcal{C}$ contains the cross-correlations between modes. Equivalently, the $\mathrm{CF}$ can be written as the complex-valued Gaussian function

$$
\begin{aligned}
& \chi\left(\lambda_{1}, \lambda_{2}\right)=\exp \left[-\left(A_{1}+\frac{1}{2}\right)\left|\lambda_{1}\right|^{2}-\frac{1}{2} B_{1}^{*} \lambda_{1}^{2}-\frac{1}{2} B_{1}\left(\lambda_{1}^{*}\right)^{2}\right] \\
& \times \exp \left[-\left(A_{2}+\frac{1}{2}\right)\left|\lambda_{2}\right|^{2}-\frac{1}{2} B_{2}^{*} \lambda_{2}^{2}-\frac{1}{2} B_{2}\left(\lambda_{2}^{*}\right)^{2}\right] \\
& \times \exp \left[-F \lambda_{1}^{*} \lambda_{2}-F^{*} \lambda_{1} \lambda_{2}^{*}+G \lambda_{1}^{*} \lambda_{2}^{*}+G^{*} \lambda_{1} \lambda_{2}\right]
\end{aligned}
$$

leading to useful expressions of the entries of the covariance matrix $\mathcal{V}$ in terms of the parameters $A_{1}, A_{2}, B_{1}, B_{2}, F, G$ 27]. After going through this correspondence we have finally obtained the $2 \times 2$ covariance matrix of the one-mode Gaussian state $M$ via Eq. (2.18),

$$
\mathcal{V}_{M}=\left(\begin{array}{ll}
\sigma(q q) & \sigma(q p) \\
\sigma(q p) & \sigma(p p)
\end{array}\right)
$$

The entries of the covariance matrix $\mathcal{V}_{M}$ are

$$
\sigma(q q)=\frac{1}{2}+\sigma\left(q_{2} q_{2}\right)+(\tan \theta)^{2} \sigma\left(q_{1} q_{1}\right)-2 \tan \theta \sigma\left(q_{1} q_{2}\right)
$$

$\sigma(q p)=\sigma\left(q_{2} p_{2}\right)-\sigma\left(q_{1} p_{1}\right)+\cot \theta \sigma\left(q_{2} p_{1}\right)-\tan \theta \sigma\left(q_{1} p_{2}\right)$ 
$\sigma(p p)=\frac{1}{2}+\sigma\left(p_{2} p_{2}\right)+(\cot \theta)^{2} \sigma\left(p_{1} p_{1}\right)+2 \cot \theta \sigma\left(p_{1} p_{2}\right)$

It is interesting to rewrite Eqs. (3.5) - 3.7 by introducing two commuting operators as combinations of the canonical operators of the two-mode resource field

$$
\hat{Q}:=\cos \theta \hat{q}_{2}-\sin \theta \hat{q}_{1}, \quad \hat{P}:=\sin \theta \hat{p}_{2}+\cos \theta \hat{p}_{1} .
$$

The operators $\hat{Q}, \hat{P}$ are closely related to those measured by Alice, Eq. (2.3). We have

$$
\begin{aligned}
& \hat{Q}\left(\hat{q}_{1}, \hat{q}_{2}\right)=\hat{q}\left(\hat{q}_{A} \rightarrow \hat{q}_{1}, \hat{q}_{i n} \rightarrow \hat{q}_{2}\right) \\
& \hat{P}\left(\hat{p}_{1}, \hat{p}_{2}\right)=\hat{p}\left(\hat{p}_{A} \rightarrow \hat{p}_{1}, \hat{p}_{i n} \rightarrow \hat{p}_{2}\right)
\end{aligned}
$$

Equations (3.5) - 3.7 become

$$
\begin{gathered}
\sigma(q q)=\frac{1}{2}+\frac{1}{(\cos \theta)^{2}}\left\langle\hat{Q}^{2}\right\rangle \\
\sigma(q p)=\frac{2}{\sin (2 \theta)}\langle\hat{Q} \hat{P}\rangle \\
\sigma(p p)=\frac{1}{2}+\frac{1}{(\sin \theta)^{2}}\left\langle\hat{P}^{2}\right\rangle .
\end{gathered}
$$

The expressions (3.10)- 3.12 allow us to enforce the interpretation given to the function (2.18) by checking on the Robertson-Schrödinger uncertainty relation

$$
\operatorname{det} \mathcal{V}_{M}=\sigma(q q) \sigma(p p)-(\sigma(q p))^{2} \geq \frac{1}{4}
$$

As we always have

$$
\left\langle\hat{Q}^{2}\right\rangle\left\langle\hat{P}^{2}\right\rangle-\langle\hat{Q} \hat{P}\rangle^{2} \geq 0,
$$

the uncertainty relation (3.13) is verified and hence the measurement-induced $M$-state is a physical state. Moreover, from Eqs. (3.10) - (3.12) we can readily find that

$$
\mathcal{V}_{M}-\frac{1}{2} I_{2} \geq 0
$$

where $I_{2}$ is the $2 \times 2$ identity matrix. The semipositiveness condition (3.15) defines the classicality of the one-mode Gaussian state $M$. This result is independent of the properties of the two-mode resourse state such as entanglement or EPR-correlations, being a consequence of the reduction of the three-mode quantum state by the measurement process.

\section{THE QUALITY OF THE TELEPORTATION PROCESS}

Following Refs. 17, 18, 20] we evaluate the teleportation quality in terms of the mean occupancy in the remote field $M$ which can be seen as the amount of noise distorting the properties of the input field state. We have

$$
N_{\text {added }}=\frac{1}{2}[\sigma(q q)+\sigma(p p)-1],
$$

and further

$$
N_{\text {added }}=\frac{1}{2}\left[\frac{1}{(\cos \theta)^{2}}\left\langle\hat{Q}^{2}\right\rangle+\frac{1}{(\sin \theta)^{2}}\left\langle\hat{P}^{2}\right\rangle\right] .
$$

From the equivalent expression

$$
\begin{aligned}
N_{\text {added }}= & \frac{1}{2}\left[\sigma\left(q_{2} q_{2}\right)+(\tan \theta)^{2} \sigma\left(q_{1} q_{1}\right)+\sigma\left(p_{2} p_{2}\right)\right. \\
& +(\cot \theta)^{2} \sigma\left(p_{1} p_{1}\right)+2 \cot \theta \sigma\left(p_{1} p_{2}\right) \\
& \left.-2 \tan \theta \sigma\left(q_{1} q_{2}\right)\right]
\end{aligned}
$$

we learn that the unbalanced measurement acts like a supplementary local squeezing of the mode 1 . We can write

$$
(\tan \theta) \hat{q}_{1} \longrightarrow{\hat{q^{\prime}}}_{1}{ }_{1}, \quad(\cot \theta) \hat{p}_{1} \longrightarrow{\hat{p^{\prime}}}_{1}
$$

and reduce the discussion to the balanced beam splittercase. It is thus sufficient to consider the balanced case when studying the optimization possibilities of the teleportation process. According to Eq. (4.3) the added noise is determined by the correlation properties of the twomode state $A B$. We denote by $b_{1}, b_{2}, c, d$ the parameters in the standard form I of the covariance matrix of a class of two-mode Gaussian states locally related by squeezing operations and having thus the same entanglement [29]. We have to minimize the added noise, Eq. (4.3) written for $\theta=\pi / 4$ and under local squeezing operations denoted by $u_{1}$ and $u_{2}$ performed on the standard resource state $\rho_{A B}^{(0)}[30]$. We write the two-variable function

$$
\begin{aligned}
N\left(u_{1}, u_{2}\right):= & \frac{1}{2}\left[b_{1}\left(u_{1}+\frac{1}{u_{1}}\right)+b_{2}\left(u_{2}+\frac{1}{u_{2}}\right)\right. \\
& \left.-2\left(c \sqrt{u_{1} u_{2}}+\frac{|d|}{\sqrt{u_{1} u_{2}}}\right)\right] .
\end{aligned}
$$

Minimization of Eq. (4.5) with respect to $u_{1}$ and $u_{2}$ leads to an algebraic system whose solution is denoted by $\vec{v}$ : $v_{1}, v_{2}$ :

$$
\begin{gathered}
\frac{b_{1}\left(v_{1}^{2}-1\right)}{v_{1}}=\frac{b_{2}\left(v_{2}^{2}-1\right)}{v_{2}} \\
b_{1} b_{2}\left(v_{1}^{2}-1\right)\left(v_{2}^{2}-1\right)=\left(c v_{1} v_{2}-|d|\right)^{2} .
\end{gathered}
$$

The general solution of this system is complicated, arising finally from a fourth-order one-variable algebraic equation. Note that Eq. (4.7) coincides with one of the equations defining the standard form II of the covariance matrix in Ref. 29], while Eq. (4.6) is different from the corresponding one first written in Ref. 29]. Now we have to recall that the standard form II of the covariance matrix 
was used by Duan et al. to give an separability criterion for two-mode Gaussian states based on the minimization of the correlations of two ingeniously defined EPR-like operators $P( \pm 1, \alpha), Q(\alpha)$. Generally, these are non-commuting operators with the notable exception of the symmetric states for which they are proportional to our operators $P$ and $Q$, Eqs. (3.8) for $\theta=\pi / 4$. Note that symmetric Gaussian states, $b_{1}=b_{2}$, have equal marginal purities. The algebraic system (4.6) - 4.7 has the unique solution

$$
v_{1}=v_{2}=\sqrt{\frac{b_{1}-|d|}{b_{1}-c}} .
$$

The squeeze factor (4.8) determines also the standard form II for which the minimal EPR correlations in the symmetric case are 31]

$$
\Delta_{E P R}=2\left[\left(b_{1}-c\right) v_{1}+\left(b_{1}-|d|\right) / v_{1}\right]=4 \tilde{c}_{-} .
$$

Here $\tilde{c}_{-}:=\sqrt{\left(b_{1}-|d|\right)\left(b_{1}-c\right)}$ is the smallest symplectic eigenvalue of the partially transpose covariance matrix of the symmetric state. According to the inseparability criterion formulated in Ref. 29], a symmetric state is entangled iff $\Delta_{E P R}<2$ namely $\tilde{c}_{-}<\frac{1}{2}$. This induces a condition to the minimal added noise obtained with the solution (4.8) for a symmetric entangled state

$$
N_{\min }\left(v_{1}, v_{1}\right)=2 \sqrt{\left(b_{1}-|d|\right)\left(b_{1}-c\right)}<1 \text {. }
$$

Therefore, we can see that an entangled Gaussian state used as a resource state in teleportation provides less noise in the output state. However, only for symmetric states a direct relation between the amount of entanglement 32 of the resource state and the quality of the teleportation could be established.

To conclude, it appears that a correspondence between the optimal added noise and the entanglement of the two-mode resource state is unlikely to be found in general. This happens because, according to the criterion in Ref. 29], the inseparability is determined by the correlations of EPR-like defined operators which are noncommuting, while the teleportation fidelity depends on correlations of typical (commuting) EPR operators. Interestingly, we have found that in the symmetric case, the minimal added noise is realized for states having the covariance matrix in standard form II [29].

\section{Acknowledgments}

This work was supported by the Romanian CNCSIS through the grant 263/2004 for the University of Bucharest.
[1] C.H. Bennett, G. Brassard, C. Crépeau, R. Jozsa, A. Peres, and W.K. Wootters, Phys. Rev. Lett. 70, 1895 (1993).

[2] A. Einstein, B. Podolsky, and N. Rosen, Phys. Rev. 47, 777 (1935).

[3] S. L. Braunstein and H. J. Kimble, Phys. Rev. Lett. 80, 869 (1998); A. Furusawa et al., Science 282, 706 (1998).

[4] S. J. van Enk, Phys. Rev. A 60, 5095 (1999).

[5] G. J. Milburn and S. L. Braunstein, Phys. Rev. A 60, 937 (1999).

[6] T. Opatrný, G. Kurizki, and D.-G. Welsch, Phys. Rev. A 61, 032302 (2000).

[7] A. Vukics, J. Janszky, and T. Kobayashi, Phys. Rev. A 66, 023809 (2002).

[8] H. F. Hofmann, T. Ide, T. Kobayashi, and A. Furusawa, Phys. Rev. A 62, 062304 (2000); T. Ide, H. F. Hofmann, A. Furusawa, and T. Kobayashi, ibid. 65, 062303 (2000).

[9] S. Scheel, L. Knöll, T. Opatrný, and D.-G. Welsch, Phys. Rev. A 62, 043803 (2000); J. Lee, M.S. Kim and, H. Jeong Phys. Rev. A 62, 032305 (2000); M.S. Kim and J. Lee, Phys. Rev. A 64, 012309 (2001); A. V. Chizhov, L. Knöll, and D.-G. Welsch, Phys. Rev. A 65, 022310 (2002); S. Oh, S. Lee, and H-w. Lee, Phys. Rev. A 66, 022316 (2002).

[10] M. Takeoka, M. Ban, and M. Sasaki, J. Opt. B: Quantum Semiclass. Opt. 4, 114 (2002).

[11] Paulina Marian, T. A. Marian, and H. Scutaru,
Rom. J. Phys. 48,727(2003). See also the e-print quant-ph/0601045 Equation 2.13) was derived here in the case of a balanced homodyne measurement, $\theta=\pi / 4$.

[12] Paulina Marian, T. A. Marian, and H. Scutaru, Phys. Rev. A 68, 062309 (2003).

[13] M. G. A. Paris, M. Cola, and R. Bonifacio, J. Opt. B: Quantum Semiclass. Opt. 5, S360 (2003).

[14] C. M. Caves and K. Wodkiewicz, Phys. Rev. Lett. 93, 040506 (2004).

[15] S. Pirandola and S. Mancini, quant-ph/0604027

[16] S. L. Braunstein and P. Van Loock, Rev. Mod. Phys. 77, $513(2005)$

[17] J. Fiurasek, Phys. Rev. A 66, 012304 (2002).

[18] L. Mista Jr and R. Filip, Phys. Rev. A 71, 032342 (2005).

[19] G. Adesso and F. Illuminati, Phys. Rev. Lett. 95, 150503 (2005).

[20] D. B. Horoshko and S. Ya. Kilin, Phys. Rev. A 61, 032304 (2000).

[21] M. M. Wolf, D. Pérez-García, and G. Giedke, quant-ph/0606132

[22] U. Leonhardt, Phys. Rev. A 48, 3265 (1993). See Eq. (39). It can be directly checked that Eq. 2.5 is a commun eigenfunction for the commuting operators (2.4).

[23] R. J. Glauber, Phys. Rev. 131,2766 (1963). Note also that when the field (2) is in a $P$-representable Gaussian state, Eq. 2.15 is the much recently studied Gaussian noise mapping. 
[24] M. S. Kim and N. Imoto, Phys. Rev. A 52, 2401 (1995).

[25] Paulina Marian and T. A. Marian, J. Phys. A: Math. Gen. 29, 6233 (1996).

[26] Paulina Marian and T. A. Marian, Phys. Lett. A 230, 276 (1997).

[27] Paulina Marian, T. A. Marian, and H. Scutaru, J. Phys A 34, 6969 (2001).

[28] M. Ban, Phys. Rev. A 69, 054304(2004).

[29] Lu-Ming Duan, G. Giedke, J. I. Cirac, and P. Zoller, Phys. Rev. Lett. 84, 2722 (2000).
[30] The standard state $\rho_{A B}^{(0)}$ is defined as the two-mode Gaussian state whose covariance matrix is in the standard form I introduced in Ref. [29]; its one-mode reductions are thermal states.

[31] Paulina Marian and T. A. Marian, to be published.

[32] G. Giedke, M. M. Wolf, O. Krüger, R. F. Werner, and J. I. Cirac, Phys. Rev. Lett. 91, 107901 (2003). In this paper it is shown that the entanglement of formation for a symmetric Gaussian state is a function of only $\tilde{c}_{-}$. 